\title{
Atividade de Culicidae em remanescente florestal na região urbana de Curitiba (Paraná, Brasil) ${ }^{1}$
}

\author{
Andréia Aparecida Barbosa ${ }^{2}$, Mario Antonio Navarro-Silva ${ }^{3} \&$ Daniéla Calado ${ }^{2}$
}

\author{
1 Contribuição número 1399 do Departamento de Zoologia, Universidade Federal do Paraná. \\ 2 Programa de Pós-graduação em Entomologia, Departamento de Zoologia, Universidade Federal do Paraná. Bolsista do \\ CNPq. Caixa Postal 19020, 81531-980 Curitiba, Paraná, Brasil. E-mail: deiaguel@bio.ufpr.br, dcalado@bio.ufpr.br \\ ${ }^{3}$ Departamento de Zoologia, Universidade Federal do Paraná. Caixa Postal 19020, 81531-980 Curitiba, Paraná, Brasil. \\ E-mail: mnavarro@bio.ufpr.br
}

\begin{abstract}
Culicidae activity in a restrict forest inside Curitiba urban area (Paraná, Brazil). Vegetation islands inside urban area can propitiate the formation of: a) adults refuge to many mosquitoes species, b) diversity of hosters, c) allows the proliferation of immature stages through naturals and artificial breeding, these ones were done by the interaction between antropic activity developed in these spaces or around areas. Considering the possibilities of contacts between the vector and the human population, the aim of this work was to verify the Culicidae species that can use man as blood source in the restricted areas of Botanical Garden, Curitiba. The capture of adults using the method of human bait at soil level, inside the forest, monthly during the period of October $13^{\text {th }} 1998$ to September $22^{\text {nd }} 1999$, from 5:00 p.m. to 8:00 p.m. 312 Culicidae specimens were captured, and 15 species were found. The predomiant species belong to Culex (Culex) coronator Dyar \& Knab, 1906 group. Others species found with potential epidemiological importance were: Haemagogus (Conopostegus) leucocelaenus (Dyar \& Shannon, 1924), Ochlerotatus (Ochlerotatus) fluviatilis (Lutz, 1904) e Anopheles (Nyssorhynchus) strodei Root, 1926.
\end{abstract}

KEY WORDS. Antropic environment, Culicidae, human bait.

A área urbana constitui ecossistema antrópico, onde o grau de artificialidade atinge seu nível mais elevado, sendo caracterizada pelo afastamento e ausência de contato com o meio natural, concentração e elevada densidade populacional e pela predominância de atividade industrial e de prestação de serviço (ForatTini 1992).

Na organização dos espaços urbanos de Curitiba, foram formadas ou preservadas áreas com características naturais que oscilam de $11.582 \mathrm{~m}^{2}$ a $8.264 .316 \mathrm{~m}^{2}$, denominadas de Parques ou Bosques, totalizando vinte e seis áreas. Tais áreas apresentam características diferentes quanto às estruturas destinadas ao lazer da população, à manutenção da cobertura vegetal original e ao recebimento de novas espécies vegetais e animais. Além de permitirem melhor drenagem do solo através da formação de lagos, reduzindo os riscos de alagamentos em áreas propícias a este evento.

Estes espaços representam um grande avanço na melhoria da qualidade de vida da população. Por outro lado, podem ser exploradas por espécies de culicídeos com diferenciado grau de sinantropia e com possibilidade de interação com o homem (URbinatTi et al. 2001). As condições existentes nestas ilhas de vegetação na estrutura urbana podem propiciar a formação de refúgios para adultos de várias espécies de mosquitos e de hospedeiros para o exercício da hematofagia. Também permitem a proliferação das formas imaturas em criadouros naturais ou artificiais, gerados pela interação com a atividade antrópica desenvolvida nestes espaços ou nas proximidades. Neste grupo de dípteros, grande número de espécies possui relevante papel na transmissão de agentes patogênicos tanto ao homem quanto aos outros animais (ForaTTINI 2002).

Dada a possibilidade de encontro de tais vetores biológicos com a população humana, reside a importância dos estudos sobre a ecologia das espécies que exploram manchas de mata, sejam de caráter silvestre, domiciliado ou em vias de assumir o comportamento ambivalente. Considerando a possibilidade de contato entre vetor e população humana, este trabalho teve como objetivo verificar as espécies de culicídeos que podem utilizar o homem como fonte de alimentação sangüínea no remanescente de mata do Jardim Botânico de Curitiba, Paraná, Brasil.

\section{MATERIAL E MÉTODOS}

As atividades de capturas dos culicídeos foram realizadas em área de remanescente de mata pertencente ao Jardim Botânico, situado na região leste do município de Curitiba 
(Estado do Paraná, Brasil) totalizando $66.022 \mathrm{~m}^{2}$ de mata. O capão de mata do Jardim Botânico consiste em uma das últimas regiões próximas ao centro da cidade na qual ainda existem representantes da floresta primária remanescente do Primeiro Planalto Paranaense. O sub-bosque é denso devido à alta taxa de regeneração dos vegetais que o compõe, possuindo dois estratos nítidos: um superior com árvores entre 1,5 a $20 \mathrm{~m}$ e um inferior com formação herbácea-arbustiva não ultrapassando $1,5 \mathrm{~m}$. A camada de serrapilheira é de aproximadamente $2 \mathrm{~cm}$ e o sombreamento é constante durante todo o dia. A área está limitada por três avenidas de tráfego intenso, uma delas recebendo o movimento de saída e chegada do litoral do Estado e próxima à Rodovia BR 116; ao lado, encontra-se também uma via férrea, que permite o tráfego. (CERvi et al. 1989).

As capturas de adultos, utilizando o homem como fator de atração, foram realizadas por dois operadores distantes dois metros um do outro ao nível do solo, no interior do capão de mata do Jardim Botânico. As coletas foram realizadas, com ritmo mensal no período de 13 de outubro de 1998 a 22 de setembro de 1999, no intervalo horário das 17:00 às 20:00 h, totalizando 48 horas de capturas. Os mosquitos capturados foram agrupados em intervalos de 30 minutos.

Simultaneamente foram utilizadas duas armadilhas luminosas tipo CDC-M (modificada por NATAL et al. 1991). As armadilhas foram instaladas no interior da mata, a aproximadamente $30 \mathrm{~m}$ da borda e distantes $3 \mathrm{~m}$ uma da outra, porém, em estratificações verticais diferentes, a 1,5 m de altura do solo e a na altura da copa das árvores. Estas armadilhas estavam distantes aproximadamente $40 \mathrm{~m}$ do local das capturas utilizando o homem como fator de atração.

Os exemplares coletados foram depositados na Coleção de Entomologia Pe. J.S. Moure (DZUP) do Departamento de Zoologia da Universidade Federal do Paraná.

Para o estudo da influência das variáveis ambientais, temperatura, precipitação pluviométrica e umidade relativa do ar sobre a atividade dos Culicidae foram obtidas planilhas de observações meteorológicas junto à Estação número 83842 do Instituto Nacional de Meteorologia, localizada no Centro Politécnico da Universidade Federal do Paraná. Esta estação situa-se nas coordenadas $25^{\circ} 25^{\prime} 00^{\prime \prime} \mathrm{S}$ e $49^{\circ} 15^{\prime} 00^{\prime \prime} \mathrm{W}$, a uma altitude de 923,50 m e distante aproximadamente $1.000 \mathrm{~m}$, na direção leste, em linha reta do Jardim Botânico. Foram coletados os dados meteorológicos referentes aos cinco dias anteriores, mais o dia efetivo de cada coleta e partir destes foram calculados os dados climáticos apresentados neste trabalho, temperaturas mínima $\left({ }^{\circ} \mathrm{C}\right)$, média e máxima, umidade relativa do ar (\%) e precipitação pluviométrica $(\mathrm{mm})$.

\section{RESULTADOS E DISCUSSÃO}

Através das capturas utilizando o homem como fator de atração, detectou-se a presença de 15 espécies em 312 exemplares pertencentes às subfamílias Culicinae e Anophelinae, com predomínio de Culex (Culex) grupo coronator, sendo cada uma das demais espécies capturadas representadas por menos de duas dezenas de exemplares (Tab. I).

No método de captura com armadilhas CDC-M, foram coletados apenas 22 exemplares de Culicidae, sendo uma espécie diferente das que ocorreram na isca humana, Mansonia (Mansonia) titillans (Walker, 1848). Os culicídeos capturados com auxílio deste método não foram considerados na apresen- tação dos resultados numéricos, assim como para confecção das tabelas I e II, devido ao número reduzido de exemplares coletados.

Tabela I. Número de exemplares e porcentagem relativa de ocorrência de espécies de Culicidae coletadas no interior de remanescente de mata do Jardim Botânico de Curitiba, através de técnica de captura humana, durante o período de 13 outubro de 1998 a 22 de setembro 1999.

\begin{tabular}{|c|c|c|}
\hline Espécies & N & $\mathrm{N} \%$ \\
\hline${ }^{*}$ Culex (Culex) grupo coronator Dyar \& Knab, 1906 & 222 & 71,2 \\
\hline Culex (Culex) spp. & 18 & 5,8 \\
\hline *Ochlerotatus (Ochlerotatus) fluviatilis (Lutz, 1904) & 16 & 5,1 \\
\hline $\begin{array}{l}\text { Haemagogus (Conospotegus) leucocelaenus (Dyar \& Shannon, } \\
\text { 1924) }\end{array}$ & 14 & 4,5 \\
\hline *Ochlerotatus (Ochlerotatus) crinifer (Theobald, 1903) & 9 & 2,9 \\
\hline Culex (Melanoconion) spp. & 5 & 1,6 \\
\hline Culex (Culex) eduardoi Casal \& Garcia, 1968 & 5 & 1,6 \\
\hline Mansonia (Mansonia) pessoai (Barreto \& Coutinho, 1944) & 4 & 1,3 \\
\hline Psorophora (Janthinosoma) ferox (Humboldt, 1819) & 3 & 1,0 \\
\hline *Trichoprosopon pallidiventer (Lutz, 1905) & 3 & 1,0 \\
\hline Mansonia (Mansonia) wilsoni (Barreto \& Coutinho, 1944) & 3 & 1,0 \\
\hline Wyomyia (Phoniomyia) quasilongirostris (Theobald, 1907) & 2 & 0,6 \\
\hline Culex (Culex) declarator Dyar \& Knab, 1906 & 2 & 0,6 \\
\hline Mansonia (Mansonia) sp. & 2 & 0,6 \\
\hline *Mansonia (Mansonia) indubitans Dyar \& Shannon, 1925 & 1 & 0,3 \\
\hline Anopheles(Nyssorhynchus) strodei Root, 1926 & 1 & 0,3 \\
\hline Ochlerotatus (Ochlerotatus) serratus (Theobald, 1901) & 1 & 0,3 \\
\hline Sabethes (Sabethes) purpureus (Theobald, 1907) & 1 & 0,3 \\
\hline Total & 312 & 100 \\
\hline
\end{tabular}

(*) Espécies capturadas com auxílio de armadilha CDC-M em dois estratos verticais.

No método utilizando o homem como fator de atração foi obtida uma amostra da diversidade das espécies presentes na área do remanescente de mata do Jardim Botânico. Neste procedimento foi obtida maior diversidade de espécies em comparação as armadilhas CDC-M. Dentre as espécies coletadas, Culex (Culex) grupo coronator destacou-se por constituir a maior parte dos exemplares capturados $(71,2 \%)$, associada à maior frequência de ocorrência durante o período estudado, com presença observada em quase todas as coletas. (Tab. II). Maiores quantidades de exemplares foram obtidas nos meses de novembro a março, período no qual foram verificadas as temperaturas mais elevadas (Fig. 1).

Cabe destacar duas espécies, Ochlerotatus fluviatilis (Lutz, 1904) e Haemagogus leucocelaenus (Dyar \& Shannon, 1924), embora coletadas em número reduzido, suas presenças foram observadas em respectivamente $58 \%$ e $41 \%$ das coletas realizadas. 
Tabela II. Ocorrência das espécies de Culicidae coletadas durante os meses no interior de remanescente de mata do Jardim Botânico de Curitiba, através de técnica de captura humana, durante o período de outubro de 1998 a de 1999.

\begin{tabular}{|c|c|c|c|c|c|c|c|c|c|c|c|c|}
\hline Espécies & Out & Nov & Dez & Jan & Fev & Mar & Abr & Mai & Jun & Jul & Ago & Set \\
\hline Culex grupo coronator & + & + & + & + & + & + & + & + & + & - & + & - \\
\hline Culex (Culex) spp. & - & + & + & + & + & + & + & + & + & - & - & - \\
\hline Ochlerotatus fluviatilis & - & - & + & + & + & + & + & + & - & + & - & - \\
\hline Haemagogus leucocelaenus & + & + & + & - & + & + & - & - & - & - & - & - \\
\hline Mansonia pessoai & + & - & + & - & - & + & + & - & - & - & - & - \\
\hline Culex eduardoi & - & + & + & - & - & - & + & - & - & - & - & - \\
\hline Culex (Melanoconion) spp. & - & + & + & - & + & - & - & - & - & - & - & - \\
\hline Ochlerotatus crinifer & + & + & + & - & - & - & - & - & - & - & - & - \\
\hline Psorophora ferox & + & - & - & + & - & + & - & - & - & - & - & - \\
\hline Trichoprosopon pallidiventer & - & + & + & - & - & + & - & - & - & - & - & - \\
\hline Mansonia (Mansonia) sp. & - & - & - & - & - & + & + & - & - & - & - & - \\
\hline Mansonia wilsoni & - & - & - & - & - & + & + & - & - & - & - & - \\
\hline Culex declarator & - & - & - & - & - & - & + & - & - & - & - & - \\
\hline Wyeomyia. quasilongirostris & - & + & - & - & - & - & - & - & - & - & - & - \\
\hline Mansonia indubitans & + & - & - & - & - & - & - & - & - & - & - & - \\
\hline Anpheles strodei & - & + & - & - & - & - & - & - & - & - & - & - \\
\hline Ochlerotatus serratus & - & + & - & - & - & - & - & - & - & - & - & - \\
\hline Sabethes purpureus & - & - & + & - & - & - & - & - & - & - & - & - \\
\hline
\end{tabular}

(+) Ocorrência, (-) não ocorrência.

Os valores de correlação entre a média de culicídeos capturados e as médias das temperaturas mínima e máxima, embora não significativos, foram mais elevados que os observados para às demais variáveis testadas. Através da análise de correlação foram verificados os seguintes valores: média da temperatura mínima (0.80), média da temperatura média (0.66), média da temperatura máxima $(0.77)$, precipitação pluviométrica acumulada $(0.27)$, média da umidade relativa mínima (0.18) e média da umidade relativa máxima (0.22).

A área do Jardim Botânico está sujeita a vários fatores interferentes, ambientais ou exercidos diretamente pelo homem, tendo em vista sua localização em zona urbana de grande movimento (CERVI et al. 1989). Constitui ambiente ambíguo por apresentar recursos naturais envolvidos pela estrutura urbana e, apesar das alterações expressivas observadas no conjunto vegetal, abriga culicídeos com intensidade diferenciada de adaptação ao ambiente e com diferentes hábitos quanto à utilização de recursos.

Apesar da menor extensão do remanescente vegetal e da possível menor oferta de criadouros, a área do Jardim Botânico de Curitiba, apresentou neste trabalho um número de espécies de Culicidae comparável a de áreas maiores, como por exemplo em trabalhos realizados em outros parques do mesmo município.

$\mathrm{Na}$ área do estudo são encontrados criadouros naturais, como ocos de árvore, lagos com vegetação aquática, poças de caráter semipermanente, bromeliáceas, entre outros. Dentre os criadouros artificiais, pode-se citar os bebedouros para animais silvestres. Estando a área do parque situada dentro do perímetro urbano e sendo circundada por habitações, estabelecimentos comerciais e terrenos em desuso, pode-se supor a existência de mais uma série de criadouros artificiais, que vêm a somar-se aos já existentes na área do capão de mata. Por outro lado, deve-se avaliar a possibilidade da área de mata vir a abrigar mosquitos de outras áreas, mesmo circunvizinhas, representando para estes um local de refúgio, além da oferta de fontes alimentares, tanto na forma de carboidratos como sangüínea.

Culex grupo coronator é considerado por LOURENÇo-DEOliveira et al. (1986) como grupo eclético quanto ao habitat. Entretanto, seu encontro em área urbana (SILVA \& Lozover 1998; Lopes et al. 1995) aliado à capacidade em explorar criadouros

Revista Brasileira de Zoologia 20 (1): 59-63, março 2003 

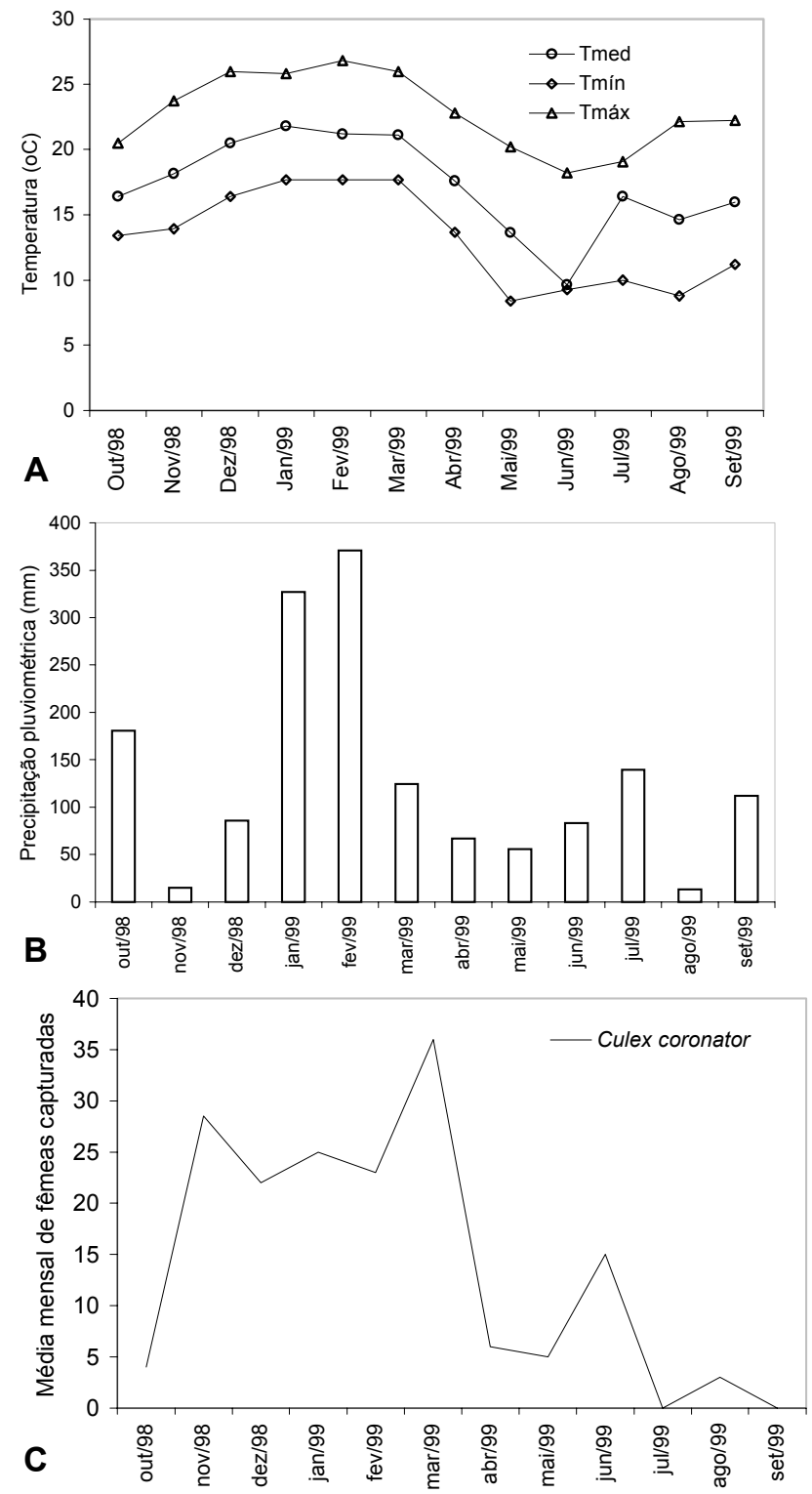

Figura 1. (A) Temperatura, (B) precipitação pluviométrica, (C) distribuição sazonal de Culex grupo coronator no período de 13 de outubro de 1998 a 22 de setembro de 1999.

artificiais (SILva \& Lozover 1996; CaLAdo \& SiLva 2001), pode indicar facilidade de adaptação ao ambiente alterado.

As espécies de Sabethini e Haemagogus leucocelaenus habitam preferencialmente o ambiente florestal e são consideradas espécies de hábitos silvestres. Segundo ForatTinI \& GoMEs (1988), Haemagogus leucocelaenus pode ser encontrada em manchas residuais de mata, as quais podem formar abrigo para esta espécie. Em área urbana da cidade de Curitiba, SiLva \& Lozover (1996) verificaram a ocorrência desta espécie, em oco de árvore, durante todo o ano.

Apesar do número reduzido de espécimens de Haema- gogus leucocelaenus coletados, eles estiveram presentes em praticamente metade das coletas. Levando-se em consideração a não utilização do método mais adequado de coleta para esta espécie e assumindo que ela não utiliza criadouro de origem antrópica, o encontro desta espécie reveste-se de significado epidemiológico, pois é considerada vetora potencial do vírus da febre amarela silvestre e está presente em diferentes regiões da área urbana de Curitiba (SiLva \& Lozovei 1999).

As espécies de Mansonia Blanchard, 1901 podem ocorrer em área urbana, entretanto sua ocorrência está sempre associada à presença de criadouros naturais com vegetação aquática. $\mathrm{Na}$ área estudada, existem lagos com vegetação aquática, representada principalmente por Eichhornia crassipes Solms. Possivelmente, o baixo número de exemplares coletados pertencentes a este gênero está sujeito a ausência de Pistia stratiotes L. nos lagos do Jardim Botânico. As maiores densidades de imaturos de Mansonia ocorrem nesta macrófita devido ao seu vasto sistema radicular (Lounibos et al. 1992), porém também ocorrem associados a Eichhornia crassipes.

Os imaturos das espécies de Anopheles Meigen, 1818, subgênero Nyssorhynchus Blanchard, 1902, são encontrados em coleções aquáticas no solo, sejam de caráter definitivo ou transitório, como pegadas de animais, buracos no solo e rochas, córregos e até em grandes depósitos de água, como lagos (Forattini 2002). No município de Curitiba, este é o primeiro encontro de Anopheles (Nyssorhynchus) strodei Root, 1926 coletado em hospedeiro humano. Tal espécie possui importância epidemiólogica, pois pode transmitir os agentes causadores da malária humana.

SiLva \& Lozovei (1996) destacaram que a proximidade ao ambiente alterado pode levar à gradual mudança quanto ao tipo de ambiente explorado, permitindo que espécies consideradas de caráter primitivo passem a explorar o ambiente alterado pela ação antrópica.

Apesar da importância estratégica que a preservação e a formação de ilhas de mata possuem na estruturação do espaço urbano e levando-se em consideração o encontro de espécies de Culicidae potencialmente vetoras de agentes etiológicos nestas áreas, o significado epidemiológico deve ser melhor avaliado sob a luz de uma maior série de dados populacionais. $\mathrm{O}$ monitoramento destas áreas através de diferentes métodos de coleta deve fazer parte da rotina dos órgãos de vigilância epidemiológica.

\section{AGRADECIMENTOS}

À Prof. Dra. Maria Anice Mureb Sallum (Faculdade de Saúde Pública da USP) pela confirmação das identificações e ao Sistema Meteorológico do Paraná (SIMEPAR) pelos dados meteorológicos.

\section{REFERÊNCIAS BIBLIOGRÁFICAS}

Calado, D.C. \& M.A.N. Silva. 2001. Comunidade de mosquitos (Diptera, Culicidae) em recipientes antrópicos introduzidos em área rural e urbana da região metropolitana de Curitiba, Paraná, Brasil. Revista Brasileira de Zoologia, Curitiba, 18 (Supl. 1): 51-60.

Cervi, A.C.; E.F. Paciornick; R.F. Vieira \& L.C. Marques. 1989. Espécies vegetais de um remanescente de Floresta de Araucária (Curitiba, Brasil): Estudo preliminar I. Acta Bio-

Revista Brasileira de Zoologia 20 (1): 59-63, março 2003 
lógica Paranaense, Curitiba, 18: 73-114.

ForatTINI, O.P. 1992. Ecologia, epidemiologia e sociedade. São Paulo, EDUSP, 529p. 2, 864p.

Forattini, O.P. \& A.C. Gomes. 1988. Biting activity of Aedes scapularis (Rondani) and Haemagogus mosquitoes in southern Brazil (Diptera: Culicidae). Revista de Saúde Pública, São Paulo, 22 (2): 84-93.

Lopes, J.; Oliveira; F.J. DE A. \& M.A.P. Tonon. 1995. Alterações na densidade populacional e diversidade de Culicidae (Diptera) na área urbana do Município de Londrina, Estado do Paraná, Sul do Brasil em Conseqüência de modificações ambientais. Semina Ciências Biológicas/Saúde, Londrina, 16 (2): 238243.

Lounibos, L.P.; N. Nishimura \& L.B. Dewald. 1992. Predation of Mansonia (Diptera: Culicidae) by native mosquito fish in Southern Florida. Journal of Medical Entomology, Lanhan, 29 (2): 236-241.

Lorenço-De-Oliveira, R.; R. Heyden \& T.F. Silva. 1986. Alguns aspectos da ecologia dos mosquitos (Diptera, Culicidae) de uma área de planície (Granjas, Calábria), em Jacarepaguá,
Rio de Janeiro.V. Criadouros. Memórias do Instituto Oswaldo Cruz, Rio de Janeiro, 81 (3): 265-271.

Natal, D.; D. Marucci; I.M. dos Reis \& E.A.B. Galati. 1991. Modificação da armadilha CDC com testes para a coleta de Flebotomíneos (Diptera). Revista Brasileira de Entomologia, São Paulo, 35 (4): 697-700.

SiLva, M.A.N \& A.L. Lozover. 1996. Criadouros de mosquitos (Diptera, Culicidae) introduzidos em mata preservada na área urbana de Curitiba, Paraná, Brasil. Revista Brasileira de Zoologia, Curitiba, 13 (4): 1023-1042.

1998. Mosquitos (Diptera, Culicidae) capturados com isca humana em área preservada de Curitiba, Paraná. Revista Brasileira de Zoologia, Curitiba, 15 (4): 965-976. . 1999. Ocorrência de Haemagogus (Conopostegus) leucocelaenus (Dyar \& Shannon) e Toxorhynchites (Lynchiella) theobaldi (Dyar \& Knab) em ocos de árvore em capão de mata, Curitiba, Paraná, Brasil. Revista Brasileira de Zoologia, Curitiba, 16 (Supl. 1): 257-267.

Urbinatti, P.R.; S. Sendaz \& D. Natal. 2001. Imaturos de mosquitos (Diptera: Culicidae) em parque de área metropolitana aberto à visitação pública. Revista de Saúde Pública, São Paulo, 35 (5): 461-466.

Recebido em 11.XI.2002; aceito em 21.II.2003.

Revista Brasileira de Zoologia 20 (1): 59-63, março 2003 